\title{
Determinação dos teores de sódio e potássio em refeições servidas em um restaurante universitário da região sul do Brasil
}

\author{
Determination of the sodium and potassium levels in meals served in a university \\ restaurant in the south of Brazil
}

\author{
Revenli Fernanda do Nascimento', Adriane Bonfim Gavron'1, Simone Bowles', Eduardo Sidinei Chaves², \\ Eliana Aparecida Fagundes Queiroz Bortolozo ${ }^{1 *}$ \\ 1 Universidade Tecnológica Federal do Paraná (UTFPR), Departamento de Alimentos, Ponta Grossa/PR - Brasil \\ 2 Universidade Tecnológica Federal do Paraná (UTFPR), Departamento de Engenharia Química, Ponta Grossa/PR - Brasil
}

\section{${ }^{*}$ Corresponding Author}

Eliana Aparecida Fagundes Queiroz Bortolozo, Universidade Tecnológica Federal do Paraná (UTFPR), Departamento de Alimentos, Av. Monteiro Lobato, s/n, km 4, CEP: 84016-210, Ponta Grossa/PR - Brasil, e-mail: bortolozo@utfpr.edu.br

Cite as: Determination of the sodium and potassium levels in meals served in a university restaurant in the south of Brazil. Braz. J. Food Technol., v. 20, e2016167, 2017.

Received: Jan. 17, 2017; Accepted: Apr. 06, 2017

\section{Resumo}

O objetivo do trabalho foi determinar os teores de sódio $(\mathrm{Na})$ e potássio $(\mathrm{K})$ em refeições servidas em um restaurante universitário da região sul do Brasil, utilizando espectrometria de emissão atômica como método analítico. Foram coletadas 30 amostras de refeições do almoço e do jantar de um restaurante universitário, referentes a 131 diferentes cardápios no período de um semestre (4 meses), levando-se em conta o consumo de todos os alimentos oferecidos no cardápio, inclusive o suco e a salada temperada. Os resultados indicaram que o teor médio de $\mathrm{Na}$ em uma refeição ultrapassou o limite diário recomendado pela Organização Mundial da Saúde (OMS) em 25 das 30 amostras analisadas. Observaram-se teores maiores em refeições compostas por algum tipo de alimento industrializado, como linguiças e batata palha, ou com pratos tradicionalmente temperados, como barreado. Os menores teores de Na estavam presentes em cardápios nos quais havia batata frita ou ovo. Os teores de K encontrados em 19 refeições poderiam ser considerados satisfatórios segundo a recomendação da OMS, no entanto, levando-se em conta que o consumo de K deve ser 1,75 vezes maior que o de $\mathrm{Na}$ em uma mesma refeição, a oferta de $\mathrm{K}$ foi baixa em todas as amostras analisadas.

Palavras-chave: Sódio; Potássio; Refeições; Restaurante universitário.

\section{Abstract}

The objective of this study was to determine the levels of sodium $(\mathrm{Na})$ and potassium $(\mathrm{K})$ in meals served in a university restaurant in the southern region of Brazil, using atomic emission spectrometry as the analytical method. Thirty samples of lunch and dinner meals were collected from a university restaurant, referring to 131 different menus in a single semester (four months), taking into account the consumption of all food offered on the menu, including the juice and seasoned salad. The results showed that the average $\mathrm{Na}$ content in a meal exceeded the daily limit recommended by the Word Health Organization (WHO) in 25 of the 30 samples analysed. Higher levels were observed in meals including some kind of processed food, such as sausages and potato sticks, or traditionally spiced dishes such as one known as 'barreado'. The lowest Na levels were found in menus including fried potato or egg. The K levels found in 19 meals could be considered satisfactory according to the $\mathrm{WHO}$ recommendations, but taking into account that the consumption of $\mathrm{K}$ should be 1.75 times greater than that of $\mathrm{Na}$ in the same meal, the supply of potassium was low in all the samples analysed.

Keywords: Sodium; Potassium; Meals; University restaurant. 


\section{Introdução}

A Hipertensão arterial sistêmica (HAS) é reconhecida como um dos maiores problemas de saúde do mundo, sendo caracterizada pela elevação da pressão arterial sistólica (PAS) a níveis maiores ou igual a $140 \mathrm{~mm} \mathrm{Hg}$ e uma pressão arterial diastólica (PAD) maior ou igual $90 \mathrm{mmHg}$ (IBIAPINA et al., 2013). Embora a HAS seja predominante entre adultos, a manifestação em crianças e adolescentes não pode ser ignorada. Estudos epidemiológicos revelam que cerca de $3 \%$ a $11 \%$ da população mundial possui hipertensão arterial em idade precoce (FREITAS et al., 2012; KAVEY et al., 2010). No Brasil, em 2013, a incidência de adultos hipertensos foi de $21,4 \%$, correspondente a 31,3 milhões de pessoas (IBGE, 2013).

O consumo de sódio $(\mathrm{Na})$ é um dos principais fatores para o aumento da incidência da patologia, sendo este proveniente principalmente do sal de cozinha e de condimentos à base de sal, além de alimentos industrializados. Segundo Sarno et al. (2013), a quantidade diária de Na disponível para consumo nos domicílios brasileiros é de aproximadamente 4,7 g para uma ingestão diária de $2.000 \mathrm{kcal}$, enquanto que o limite máximo recomendado pela Organização Mundial da Saúde (OMS) é de 2 g/dia (WHO, 2006).

Em contrapartida, a OMS recomenda que a ingestão diária de potássio (K) deve ser de no mínimo 3,5 g, visto que este cátion possui efeito anti-hipertensivo porque induz uma perda pronunciada de água e Na pelo corpo, realiza a supressão da secreção de renina e angiotensina, aumenta a secreção de prostaglandina, atua reduzindo a resistência vascular periférica pela dilatação arteriolar direta, diminui o tônus adrenérgico e estimula a atividade da bomba iônica de $\mathrm{Na}$, $\mathrm{K}+$-ATPase (TOMAZONI; SAVIERO, 2009).

A admissão ao ensino superior é considerada um momento de destaque na vida de adultos jovens. Pode, entretanto, desencadear mudanças significativas do estilo de vida, como a sobreposição de atividades, mudanças comportamentais, planejamento inapropriado do tempo, entre outros fatores psicossociais envolvidos. Tais condições são responsáveis também por alterações significativas nos hábitos alimentares (DUARTE et al., 2013), que, segundo Davy et al. (2006), tendem a piorar durante a permanência no ensino universitário, como a omissão de refeições e a escolha de alimentos mais calóricos e ultraprocessados.

Neste contexto, as universidades tornam-se centros importantes para a promoção de estilos de vida saudáveis dos jovens adultos, pois a situação nutricional desse grupo populacional pode trazer como consequência o aparecimento de doenças Crônicas Não Transmissíveis (DCNT), como obesidade, diabetes, hipertensão arterial sistêmica (HAS), dislipidemias, entre outras (FREITAS et al., 2012).
Há uma lacuna na literatura, no tocante ao teor de $\mathrm{Na}$ e K em refeições servidas em restaurantes universitários, determinados a partir de métodos instrumentais. Levando-se em conta a alta incidência na população de HAS e o papel dos restaurantes universitários na promoção de hábitos alimentares em jovens universitários, este estudo teve como objetivo determinar os teores de $\mathrm{Na}$ e $\mathrm{K}$ em refeições servidas em um restaurante universitário por espectrometria de emissão atômica.

\section{Material e métodos}

Trata-se de um estudo transversal e experimental do teor de $\mathrm{Na}$ e $\mathrm{K}$ de refeições (almoço e jantar) servidas em um restaurante universitário de uma universidade pública do Estado do Paraná, Brasil, cujos alunos frequentam cursos integrais (Engenharias) ou noturnos (Tecnologias). O restaurante fornece diariamente 700 refeições no almoço e 300 no jantar. Foram analisados os teores de $\mathrm{Na}$ e $\mathrm{K}$ dos alimentos fornecidos, nos cardápios do almoço e jantar, durante o primeiro semestre letivo do ano de 2015 (4 meses), a partir de espectrometria de emissão atômica, segundo método descrito por Dasbasi et al. (2016) e Ploegaerts et al. (2016).

\subsection{Tamanho da amostra}

O planejamento do tamanho mínimo da amostra, considerando-se 95\% de significância, foi delineado segundo metodologia de Triola (2008), para população finita, conforme a Equação 1. O tamanho da amostra $(\mathrm{N}=132)$ refere-se aos diferentes cardápios fornecidos durante o período do estudo para a comunidade acadêmica (alunos e técnicos administrativos). Tendo-se em vista minimizar erros nas estimativas médias, adotou-se um desvio padrão médio calculado com base nos resultados de Salas et al. (2009). O cálculo da amostra totalizou um número mínimo de 30 amostras.

$$
\mathrm{n}=\frac{\mathrm{N} \cdot \sigma^{2} \cdot\left(\mathrm{Z}_{\alpha / 2}\right)^{2}}{(\mathrm{~N}-1) \mathrm{E}^{2}+\sigma^{2} \cdot\left(\mathrm{Z}_{\alpha / 2}\right)^{2}}
$$

\section{Onde:}

n: tamanho da amostra

$\mathrm{N}: 132$ (tamanho da população)

б: $518 \mathrm{mg}$ (desvio padrão populacional)

E: 163,56 (margem de erro da estimativa de um parâmetro populacional)

$\mathrm{Z}_{\alpha / 2}: 1,96$ (valor crítico, relacionado com o grau de confiança adotado).

\subsection{Preparo das amostras}

Os materiais que entraram em contato direto com as amostras foram desmineralizados em banho de ácido nítrico $\left(\mathrm{HNO}_{3}\right)$ 10\% v/v por 12 horas, utilizando para 
limpeza água ultrapura com resistividade de $18 \mathrm{M} \Omega \mathrm{cm}$ (Master System MS 2000, GEHAKA).

Todos os constituintes da porção coletada foram homogeneizados em liquidificador caseiro e armazenados em temperatura de congelamento $\left(-6^{\circ} \mathrm{C}\right)$, até a realização das análises de $\mathrm{Na}$ e $\mathrm{K}$.

\subsection{Análise de Na e K}

Depois do descongelamento das amostras, essas foram submetidas à digestão ácida por via úmida. Aproximadamente $1 \mathrm{~g}$ de cada amostra foi pesado em balança analítica diretamente em tubos de Kjeldahl, nos quais foram adicionados $4 \mathrm{~mL}$ de $\mathrm{HNO}_{3} 65 \%$ (Merck) e $2 \mathrm{~mL}$ de peróxido de hidrogênio 30\% suprapuro (Vetec) e, posteriormente, foram submetidas a aquecimento em bloco digestor modelo SL 25/40 (SOLAB), à temperatura de $80{ }^{\circ} \mathrm{C}$ por 30 minutos e, em seguida, a $120{ }^{\circ} \mathrm{C}$ por mais 90 minutos.

Depois da digestão, as amostras foram filtradas e avolumadas para $50 \mathrm{~mL}$ com água ultrapura. A determinação das concentrações dos elementos foi realizada por espectrometria de emissão atômica, modelo $610 \mathrm{MS}$ (Analyser), sendo que as amostras de branco (4 mL de $\mathrm{HNO}_{3}$ 65\% e $2 \mathrm{~mL}$ peróxido de hidrogênio 30\%) foram analisadas paralelamente. A análise de cada amostra foi realizada em triplicata.

\subsection{Análise dos dados}

Os resultados obtidos para Na e K foram comparados com a recomendação do Guia Alimentar para a População Brasileira (BRASIL, 2006), que estabelece o consumo destes nutrientes para uma única refeição, sendo recomendado 800 mg e 1.400 mg, respectivamente. Também houve a comparação com as recomendações da OMS, que estabelece o consumo diário de no máximo 2.000 mg de $\mathrm{Na}$ e o consumo mínimo de 3.500 mg de K/dia (WHO, 2006).

\subsection{Análise estatística dos resultados}

A análise estatística dos dados foi realizada através do software SASM-Agri (Sistema para análise e separação de médias em experimentos agrícolas), no qual os resultados foram submetidos à análise de variância (ANOVA) e, em seguida, ao teste de separação de Tukey para identificar diferença estatística ao nível de 5\% de significância $(p<0,05)$.

\section{Resultados e discussão}

O restaurante universitário serve uma opção proteica (carne ou ovo), arroz, feijão, guarnição, farofa, dois tipos de salada, vinagrete, molho para salada e um copo de suco. As amostras foram compostas por todos os alimentos que compõem o buffet, incluindo a salada temperada e um copo de suco.

A amostragem foi composta por $65 \mathrm{~g}$ de arroz, $50 \mathrm{~g}$ de feijão, $60 \mathrm{~g}$ de guarnição, $120 \mathrm{~g}$ de carne, $12 \mathrm{~g}$ de farofa, $12 \mathrm{~g}$ de vinagrete, $12 \mathrm{~g}$ de molho de salada, $85 \mathrm{~g}$ de salada e $250 \mathrm{ml}$ de suco, totalizando uma porção de $666 \mathrm{~g}$, sendo que a proporção de cada preparação que compôs a amostragem foi baseada nos seus per capitas, segundo valores informados pelo responsável técnico do restaurante.

Os resultados encontrados para o $\mathrm{Na}$ e $\mathrm{K}$ no restaurante em estudo são apresentados na Tabela 1.

Em ordem decrescente observou-se que os maiores teores de $\mathrm{Na}$ corresponderam às amostras 25, 30, 24 e 20, os quais não diferiram estatisticamente. O elevado teor de $\mathrm{Na}$ nessas amostras provavelmente deve-se à presença de alimentos industrializados como é o caso da batata palha e da linguiça. Outro fator considerável foi a presença do Barreado na amostra 20, prato típico do Paraná, caracterizado por seu sabor acentuado, bastante temperado. Em contrapartida, os menores teores de $\mathrm{Na}$ corresponderam às amostras 4, 12, 13 e 14, as quais não apresentaram diferença estatística entre si. O menor teor de $\mathrm{Na}$ encontrado nessas amostras deveu-se principalmente à presença do ovo como prato principal, que, além de constituir uma porção menor em relação aos demais pratos principais, não era temperado. Pode-se mencionar a presença de batata frita como guarnição em uma das amostras, à qual também não foi acrescentado sal, e a ausência de molho em duas das amostras analisadas. Uma pesquisa realizada por Borjes et al. (2014) revelou que no molho caseiro disponibilizado em restaurantes comerciais de Chapecó-SC encontravam-se 977 mg de $\mathrm{Na} / 10 \mathrm{~g}$ do alimento, e no molho rosé $769 \mathrm{mg}$ de $\mathrm{Na} / 100 \mathrm{~g}$ do alimento, demostrando o elevado teor desse nutriente em tais preparações.

Segundo a Recommended Dietary Allowances (NRC, 1989), a quantidade de $\mathrm{Na}$ total presente em uma refeição não deve ultrapassar 600 mg de $\mathrm{Na}$, e a recomendação do Guia Alimentar para a População Brasileira (BRASIL, 2006) varia entre 600 mg e 800 mg de $\mathrm{Na}$. Analisando a Tabela 1, que apresenta os teores totais de $\mathrm{Na}$ presente nas amostras, verificaram-se elevados teores de $\mathrm{Na}$, ultrapassando não somente a recomendação diária para uma refeição, como a recomendação diária da OMS, que estabelece como consumo máximo 2.000 mg de $\mathrm{Na}$ (WHO, 2006).

Resultados semelhantes também foram encontrados por Silva et al. (2014) ao estudarem dois restaurantes comerciais à La carte de uma praça de alimentação de uma Universidade da cidade de São Paulo (SP), nos quais relataram que os alimentos servidos durante três dias excederam o limite máximo de Na recomendado pelo Guia Alimentar para a População Brasileira. Salas et al. 
(2009), ao avaliar o consumo de Na em uma empresa do município de Suzano - SP, referiram uma média de consumo de Na na refeição almoço equivalente a $2.435 \mathrm{mg}$, superior à recomendação diária da OMS. Excessos de Na também foram descritos por Capalonga et al. (2010) ao determinarem a quantidade de sal oferecida no almoço dos funcionários de um hospital público de Porto Alegre, que apresentou uma média de consumo de $\mathrm{Na}$ em torno de $1.980 \mathrm{mg}$, valor correspondente a 99\% da recomendação total diária segundo a OMS (WHO, 2006).

Há evidências científicas dos efeitos benéficos da restrição no consumo de sal na dieta. Estima-se que, entre 25 e 55 anos de idade, uma diminuição de apenas 1,3 g na quantidade de $\mathrm{Na}$ consumida diariamente se traduziria em uma redução de $20 \%$ na prevalência de hipertensão arterial, bem como de outras doenças vasculares e coronarianas, representando 150 mil vidas salvas anualmente em todo o mundo. Há dez anos, o departamento de saúde canadense incentiva a população a consumir um limite máximo de 1,8g de Na por dia. Para chegar à meta, em geral, seria preciso retirar do cardápio duas colheres de sopa de sal da alimentação diária. Os dados mostraram que a redução do sal e do Na promoveu uma diminuição de $13 \%$ das mortes por doenças coronarianas, $8 \%$ do número de infartos e $12 \%$ dos acidentes vasculares cerebrais (IGNÁCIO et al., 2013).

Em relação à quantidade de $\mathrm{K}$ presente nas amostras analisadas (Tabela 1), notou-se que os maiores teores correspondem às amostras 28 e 29, bem como os menores estão presentes nas amostras 11 e 12. Essa diferença provavelmente decorreu da combinação de dois alimentos ricos em $\mathrm{K}$, a beterraba e o rabanete, visto que essa mistura

Tabela 1. Quantidade de Sódio e Potássio presente nas amostras analisadas.

\begin{tabular}{|c|c|c|c|c|}
\hline Amostra & $\begin{array}{c}\mathrm{Na} \\
(\mathrm{mg} / 100 \mathrm{~g})\end{array}$ & $\begin{array}{c}\mathrm{Na} \\
\text { (mg/refeição) }\end{array}$ & $\begin{array}{c}\mathrm{K} \\
(\mathrm{mg} / 100 \mathrm{~g})\end{array}$ & $\begin{array}{c}\text { K } \\
\text { (mg/refeição) }\end{array}$ \\
\hline 1 & $319,67 \pm 20,11^{\mathrm{jkl}}$ & $2129 \pm 134$ & $187,67 \pm 9,61^{\mathrm{fgh}}$ & $1249,88 \pm 64$ \\
\hline 2 & $409,33 \pm 16,19^{\text {bcdefg }}$ & $2726,14 \pm 107,82$ & $130,33 \pm 3,21^{1 \mathrm{~m}}$ & $868 \pm 21,38$ \\
\hline 3 & $314 \pm 15,52^{\mathrm{kl}}$ & $2091,24 \pm 103,36$ & $151,67 \pm 3,21^{1 \mathrm{kl}}$ & $1010,12 \pm 21,38$ \\
\hline 4 & $258 \pm 3^{m}$ & $1718,28 \pm 19,98$ & $208,33 \pm 1,53^{\text {def }}$ & $1387,48 \pm 10,19$ \\
\hline 5 & $397,67 \pm 5,69^{\text {defgh }}$ & $2648,48 \pm 37,89$ & $165,67 \pm 2,08^{i j k}$ & $1103,36 \pm 13,85$ \\
\hline 6 & $359 \pm 22,71^{\text {hijk }}$ & $2390,94 \pm 161,24$ & $169,33 \pm 9,24^{\text {hij }}$ & $1127,74 \pm 61,54$ \\
\hline 7 & $329 \pm 25,06^{i j k}$ & $2191,14 \pm 166,9$ & $133,33 \pm 12,74^{\mathrm{Im}}$ & $887,98 \pm 84,85$ \\
\hline 8 & $411,67 \pm 3,78^{\text {bcdef }}$ & $2741,72 \pm 25,17$ & $180,67 \pm 1,53^{\mathrm{ghi}}$ & $1203,26 \pm 10,19$ \\
\hline 9 & $374,33 \pm 4,93^{\text {efghi }}$ & $2493,04 \pm 32,83$ & $170,33 \pm 7,77^{\text {hij }}$ & $1134,4 \pm 51,75$ \\
\hline 10 & $311,33 \pm 5,03^{k l}$ & $2073,46 \pm 33,5$ & $121,67 \pm 1,53^{\mathrm{mn}}$ & $810,32 \pm 10,19$ \\
\hline 11 & $318,33 \pm 9,07^{\mathrm{ikl}}$ & $2120,08 \pm 60,41$ & $90 \pm 2,64^{\circ \mathrm{pp}}$ & $599,4 \pm 17,58$ \\
\hline 12 & $254,67 \pm 15,88^{m}$ & $1696,10 \pm 105,76$ & $79 \pm 1,73^{p}$ & $526,14 \pm 11,52$ \\
\hline 13 & $246,33 \pm 15,53^{m}$ & $1640,56 \pm 103,43$ & $103,33 \pm 6,43^{\text {no }}$ & $688,18 \pm 42,83$ \\
\hline 14 & $233,33 \pm 6,11^{m}$ & $1553,98 \pm 40,7$ & $137,33 \pm 5,58^{\mathrm{m}}$ & $914,62 \pm 37,16$ \\
\hline 15 & $407,33 \pm 2,89^{\text {cdefgh }}$ & $2712,82 \pm 19,25$ & $132,67 \pm 3,05^{\operatorname{lm}}$ & $883,58 \pm 20,31$ \\
\hline 16 & $273,67 \pm 4,04^{1 m}$ & $1822,64 \pm 26,9$ & $164,33 \pm 4,04^{\mathrm{ijk}}$ & $1094,44 \pm 26,9$ \\
\hline 17 & $365,67 \pm 11,01^{\text {fghi }}$ & $2435,36 \pm 73,33$ & $197,67 \pm 5,03^{\text {efg }}$ & $1316,48 \pm 33,5$ \\
\hline 18 & $381,67 \pm 9,61^{\text {efgh }}$ & $2541,92 \pm 64$ & $145 \pm 3,60^{\mathrm{kl}}$ & $965,7 \pm 23,98$ \\
\hline 19 & $365 \pm 8,18^{\text {tghij }}$ & $2430,9 \pm 54,48$ & $169,33 \pm 4,16^{\text {hij }}$ & $1127,74 \pm 27,71$ \\
\hline 20 & $437,33 \pm 29,74^{\mathrm{abcd}}$ & $2912,62 \pm 198,07$ & $181,67 \pm 4,04^{g h i}$ & $1209,92 \pm 26,91$ \\
\hline 21 & $384,67 \pm 31,88^{\text {efgh }}$ & $2561,90 \pm 212,32$ & $147,67 \pm 11^{\mathrm{kl}}$ & $983,48 \pm 73,26$ \\
\hline 22 & $359,67 \pm 16,19$ ghijk & $2395,40 \pm 107,8$ & $134,33 \pm 6,11^{1 \mathrm{~m}}$ & $894,64 \pm 40,79$ \\
\hline 23 & $390 \pm 26,96^{\text {defgh }}$ & $2597,4 \pm 179,55$ & $219,33 \pm 8,62^{\mathrm{bcd}}$ & $1460,74 \pm 57,41$ \\
\hline 24 & $452 \pm 19,08^{a b c}$ & $3010,32 \pm 127,07$ & $205 \pm 3,60^{\text {def }}$ & $1365,3 \pm 23,98$ \\
\hline 25 & $475 \pm 6,08^{a}$ & $3163,5 \pm 40,49$ & $234 \pm 3^{b c}$ & $1558,44 \pm 19,98$ \\
\hline 26 & $367 \pm 6,08^{\text {fghij }}$ & $2444,22 \pm 40,49$ & $239,67 \pm 13,61^{b}$ & $1596,20 \pm 90,64$ \\
\hline 27 & $380,67 \pm 18,5^{\text {efgh }}$ & $2535,26 \pm 123,21$ & $216,33 \pm 12,58^{\text {cde }}$ & $1440,76 \pm 83,78$ \\
\hline 28 & $365 \pm 15,88^{\text {fghij }}$ & $2431,89 \pm 105,76$ & $275,67 \pm 10,11^{a}$ & $1835,96 \pm 67,33$ \\
\hline 29 & $420,67 \pm 11,93^{\text {bcde }}$ & $2801,66 \pm 79,45$ & $276,67 \pm 3,21^{a}$ & $1842,62 \pm 21,38$ \\
\hline 30 & $459 \pm 11,79^{\mathrm{ab}}$ & $3056,94 \pm 78,52$ & $232,67 \pm 3,78^{\mathrm{cb}}$ & $1549,58 \pm 25,17$ \\
\hline
\end{tabular}

Refeição de $666 \mathrm{~g}$, com todos os alimentos que compõem o cardápio. Os dados mostrados representam a média de três repetições. Letras diferentes indicam diferença estatística ao nível de 5\% de significância. Recomendação Guia Alimentar (mg de Na/refeição=800; mg de K/refeição=1.400), recomendação OMS (WHO, 2006). (mg de Na/dia=2.000; mg de K/dia=3.500). 


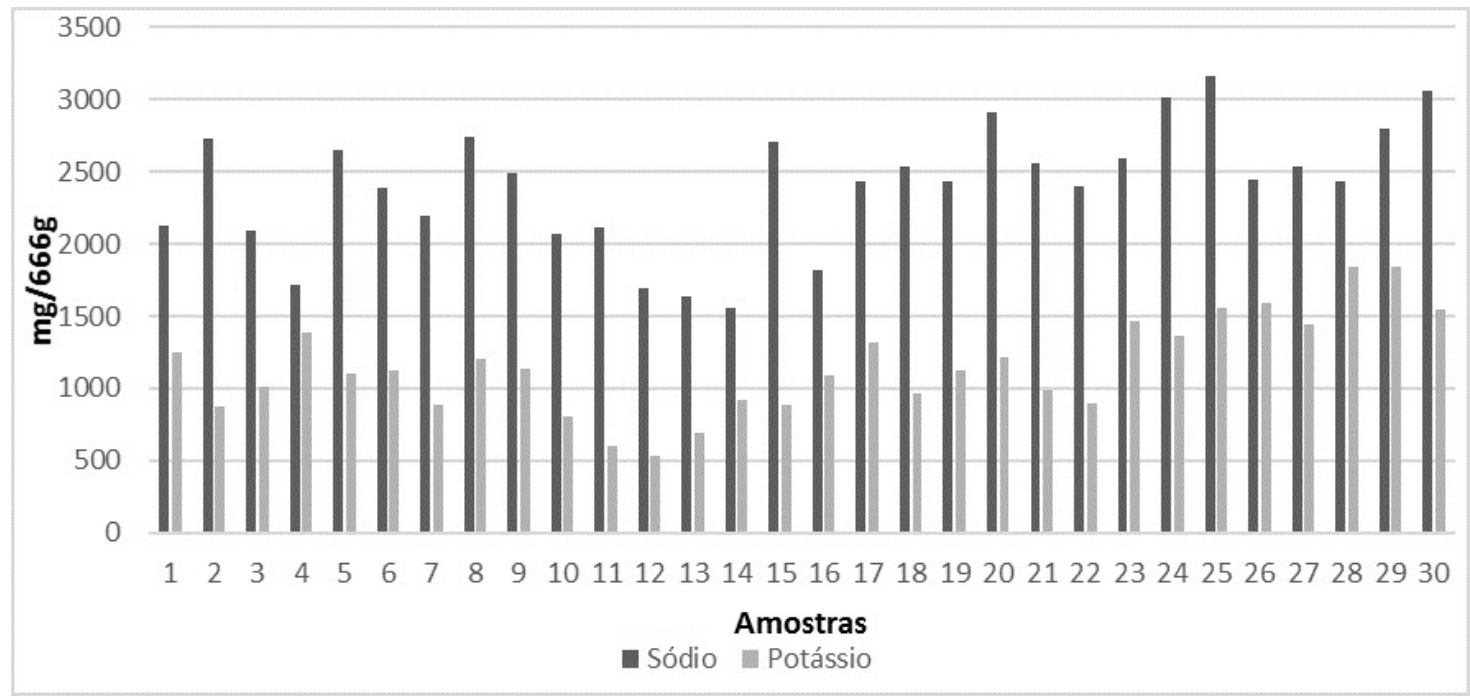

Figura 1. Relação entre Sódio e Potássio nas refeições servidas em restaurante universitário.

de salada não foi observada em nenhuma outra amostra. Segundo a tabela TACO (UNICAMP, 2011), a beterraba crua possui cerca de $375 \mathrm{mg}$ de $\mathrm{K} / 100 \mathrm{~g}$ e o rabanete cru possui $328 \mathrm{mg}$ de $\mathrm{K} / 100 \mathrm{~g}$ de alimento.

De acordo com a OMS, o consumo de $\mathrm{K}$ deve ser 1,75 vezes maior que o de $\mathrm{Na}$ em uma mesma refeição. Levando em conta essa informação e analisando a recomendação do Guia Alimentar para o teor de Na, pode-se inferir que para uma única refeição a recomendação de $\mathrm{K}$ variou entre $1.050 \mathrm{mg}$ e $1.400 \mathrm{mg}$. Observou-se que, na maioria das refeições, o consumo de K esteve dentro do recomendado pelo Guia Alimentar, permanecendo abaixo dessa recomendação em 11 refeições. No entanto, quando comparado com o elevado teor de $\mathrm{Na}$, o K apresentou proporção variando de 0,28 a 0,66, enquanto o ideal seria apresentar-se na proporção de 1,75. A Figura 1 ilustra essa grande diferença de valores entre o $\mathrm{Na}$ e o K.

Resultados semelhantes aos encontrados para o $\mathrm{K}$ neste trabalho foram relatados por Passos e Ferreira (2010), ao avaliarem a qualidade nutricional da dieta oferecida em uma instituição de longa permanência para idosos, em que foi descrita a oferta superior para o $\mathrm{Na}$ e inferior para o K, segundo as recomendações da OMS. Em todas as opções de refeições, a oferta de $\mathrm{K}$ foi abaixo da recomendação atual, entre 33\% e 52\%. Martins (2009), ao quantificar os teores de $\mathrm{Na}$ e $\mathrm{K}$ em 28 amostras de sopa, recolhidas em duas escolas, descreveu que, em todas as refeições, os teores de K obtidos estavam abaixo dos encontrados para o Na. Níveis baixos de K também foram referidos por Segalla e Spinelli (2012), em seus estudos sobre qualidade nutricional de refeições para idosos institucionalizados em uma instituição de longa permanência. Os teores de K foram 54\% a $60 \%$ abaixo do recomendado para essa classe populacional, enquanto que os níveis de Na apresentaram-se de 60\% a 68\% acima do recomendado para idosos.

Na tentativa de corrigir esse desequilíbrio na dieta, pode-se utilizar produtos comerciais com substituição do cloreto de sódio ( $\mathrm{NaCl}$ ) pelo cloreto de potássio $(\mathrm{KCl})$, o chamado "sal light", que possui teor de Na reduzido em até 60\% (FILIPINI et al., 2014), pois a substituição completa do $\mathrm{NaCl}$ pelo $\mathrm{KCl}$ não é recomendada, devido ao sabor amargo conferido aos produtos, o que geralmente o torna pouco aceitável (NASCIMENTO et al., 2007). Braschi et al. (2009) elaboraram pães brancos em que a formulação padrão com 1,8\% de $\mathrm{NaCl}$ foi substituída por sais de $\mathrm{K}$, como KCl, citrato de $\mathrm{K}$ mono-hidratado, hidrogenocarbonato de $\mathrm{K}$ e gluconato de $\mathrm{K}$, em níveis de substituição que variaram entre 20 a $75 \%$ do peso de $\mathrm{NaCl}$. Estes autores avaliaram a aceitabilidade dos pães e a biodisponibilidade dos sais de $\mathrm{K}$ adicionados e concluíram que níveis de até $30 \%$ de substituição proporcionaram escores de aceitação semelhantes à formulação padrão.

\section{Conclusão}

Os teores de $\mathrm{Na}$ encontrados foram superiores à recomendação do Guia Alimentar para a população brasileira em todas as refeições analisadas, referindo-se a uma única refeição, bem como a maioria das amostras apresentou teores de $\mathrm{Na}$ superiores à recomendação diária da OMS. Em contrapartida, os teores de K, segundo as recomendações do Guia Alimentar para a população brasileira e da OMS, estão dentro do limite diário. No entanto, quando comparados aos teores de $\mathrm{Na}$, verificou-se que a ingestão de $\mathrm{K}$ foi baixa, pois a ingestão de $\mathrm{K}$ deve ser 1,75 vezes maior que a ingestão de $\mathrm{Na}$, e a relação apresentada foi de 0,28 a 0,66. 
Determinação dos teores de sódio e potássio em refeições servidas em um restaurante universitário da região sul do Brasil Nascimento, R. F. et al.

São necessárias medidas para a redução do consumo de sal e o equilíbrio com a concentração de K nos alimentos preparados no restaurante objeto de estudo.

\section{Agradecimentos}

Agradecemos à Universidade Tecnológica Federal do Paraná pelo apoio financeiro recebido e ao restaurante universitário por ter permitido a realização deste trabalho.

\section{Referências}

BORJES, L. C.; TASCA, F. J.; ZAMPROGANA, P. E. Alimentos industrializados fontes de sódio utilizados no preparo de refeições em restaurantes comerciais de Chapecó-SC. Demetra, Rio de Janeiro, v. 9, n. 1, p. 83-97, 2014. http://dx.doi.org/10.12957/ demetra.2014.7249.

BRASCHI, A.; GILL, L.; NAISMITH, D. J. Partial substitution of sodium with potassium in white bread: feasibility and bioavailability. International Journal of Food Sciences and Nutrition, Basingstoke, v. 60, n. 6, p. 507-521, 2009. PMid:18608540. http://dx.doi.org/10.1080/09637480701782118.

BRASIL. Ministério da saúde. Secretaria de Atenção à Saúde. Coordenação Geral da Política de Alimentação e Nutrição. Guia alimentar para a população brasileira: promovendo a alimentação saudável/alimentação e nutrição. Brasília: Ministério da Saúde, 2006

CAPALONGA, R.; TONIOLO, L.; SOLTYS, A. A.; OLIVEIRA, A. B. A. Avaliação da quantidade de sal oferecida no almoço dos funcionários de um hospital público de Porto Alegre. Revista HCPA, Porto Alegre, v. 30, n. 3, p. 204-208, 2010

DASBASI, T.; SAÇMACI, S.; ÜLGEN, A.; KARTAL, S. Determination of some metal ions in various meat and baby food samples by atomic spectrometry. Food Chemistry, London, v. 197, n. Pt A, p. 107-113, 2016. PMid:26616930. http://dx.doi.org/10.1016/j. foodchem.2015.10.093.

DAVY, S. R.; BENES, B. A.; DRISKELL, J. A. Sex differences in dieting trends, eating habits, and nutrition beliefs of a group of midwestern college students. Journal of the American Dietetic Association, Bethesda, v. 106, n. 10, p. 1673-1677, 2006. PMid:17000202. http://dx.doi.org/10.1016/j.jada.2006.07.017.

DUARTE, F. M.; ALMEIDA, S. D. S.; MARTIN, K. A. Alimentação fora do domicílio de universitários de alguns cursos da área da saúde de uma instituição privada. O Mundo da Saúde, São Paulo, v. 37, n. 3, p. 288-298, 2013.

FILIPINI, K.; GOMES, C. C.; CARVALHO, A. P. P. F.; VIEIRA, L. L. Aceitação da dieta hipossódica com sal de cloreto de potássio (sal light) em pacientes internados em um hospital público. Revista de Atenção à Saúde, São Caetano do Sul, v. 12, n. 41, p. 11-18, 2014. http://dx.doi.org/10.13037/rbcs.vol12n41.2093.

FREITAS, D.; RODRIGUES, C. S.; YAGUI, C. M.; CARVALHO, R. S. T.; MARCHI-ALVES, L. M. Fatores de risco para hipertensão arterial entre estudantes do ensino médio. Acta Paulista de Enfermagem, São Paulo, v. 25, n. 3, p. 430-434, 2012. http:// dx.doi.org/10.1590/S0103-21002012000300017.

IBIAPINA, D. F. N.; SANTOS, A. N.; OLIVEIRA, L. N. R. Conhecimento dos pacientes com hipertensão arterial sobre a quantidade de sódio presente nos alimentos. Revista Interdisciplinar, Teresina, v. 6, n. 4, p. 75-85, 2013

IGNÁCIO, A. K.; RODRIGUES, J. T. D.; NIIZU, P. Y.; CHANG, Y. K.; STEEL, C. J. Efeito da substituição de cloreto de sódio por cloreto de potássio em pão francês. Brazilian Journal of Food Technology, Campinas, v. 16, n. 1, p. 1-11, 2013. http://dx.doi. org/10.1590/S1981-67232013005000010.

INSTITUTO BRASILEIRO DE GEOGRAFIA E ESTATÍSTICA - IBGE. Pesquisa nacional de saúde: percepção do estado de saúde, estilos de vida e doenças e crônicas. Rio de Janeiro, 2013

KAVEY, R. E.; DANIELS, S. R.; FLYNN, J. T. Management of high blood pressure in children and adolescents. Cardiology Clinics, Philadelphia, v. 28, n. 4, p. 597-607, 2010. PMid:20937444. http:// dx.doi.org/10.1016/j.ccl.2010.07.004.

MARTINS, M. J. R. Hábitos alimentares de estudantes universitários. 2009. 41 f. (Trabalho de Investigação)-Faculdade de Ciências da Nutrição e Alimentação, Universidade do Porto, Porto, 2009.

NASCIMENTO, R.; CAMPAGNOL, P. C. B.; MONTEIRO, E. S.; POLLONIO, M. A. R. Substituição de cloreto de sódio por cloreto de potássio: influência sobre as características físico químicas e sensoriais de salsichas. Alimentos e Nutrição, Araraquara, v. 18, n. 3, p. 297-302, 2007.

NATIONAL RESEARCH COUNCIL - NRC. Recomended dietary allowances. 10th ed. Washington: National Academic Press, 1989.

PASSOS, J. P.; FERREIRA, K. S. Caracterização de uma instituição de longa permanência para idosos e avaliação da qualidade nutricional da dieta oferecida. Alimentos e Nutrição, Araraquara, v. 21, n. 2, p. 241-249, 2010.

PLOEGAERTS, G.; DESMET, C.; VAN KRIEKEN, M. Assay of sodium in food: comparison of different preparation methods and assay techniques. Journal of Food Composition and Analysis, San Diego, v. 45, p. 66-72, 2016. http://dx.doi.org/10.1016/j. jfca.2015.09.017.

SALAS, C. K. T.; SPINELLI, M. G. N.; KAWASHIMA, L. M.; UEDA, A. M. Teores de sódio e lipídios em refeições almoço consumidas por trabalhadores de uma empresa do município de Suzano, SP. Revista de Nutrição, Campinas, v. 22, n. 3, p. 331-339, 2009. http://dx.doi.org/10.1590/S1415-52732009000300003.

SARNO, F.; CLARO, R. M.; LEVY, R. B.; BANDONI, D. H.; MONTEIRO, C. A. Estimativa de consumo de sódio pela população brasileira, 2008-2009. Revista de Saúde Pública, São Paulo, v. 47, n. 3 , p. 571-578, 2013. PMid:24346570. http://dx.doi.org/10.1590/ S0034-8910.2013047004418. 
Determinação dos teores de sódio e potássio em refeições servidas em um restaurante universitário da região sul do Brasil Nascimento, R. F. et al.

SEGALLA, R.; SPINELLI, R. B. Análise nutricional para realizar atenção a idosos de uma instituição de longa permanência, no município de Erechim-RS. Vivências, Erexim, v. 8, n. 14, p. 72-85, 2012.

SILVA, A. P.; DIAS, G. C.; ABREU, E. S.; SPINELLI, M. G. N.; MATIAS, A. C. G. Teores de sódio e lipídeos na refeição de restaurantes comerciais de uma universidade da cidade de São Paulo. Alimentos e Nutrição, Araraquara, v. 25, n. 1, p. 25-31, 2014.

TOMAZONI, T.; SAVIERO, J. Consumo de potássio de idosos hipertensos participantes do Programa Hiperdia do município de Caxias do Sul, RS. Revista Brasileira de Hipertensão, São Paulo, v. 16, n. 4, p. 246-250, 2009.

TRIOLA, M. F. Introdução à estatística. 10. ed. São Paulo: LTC, 2008. $696 \mathrm{p}$.

UNIVERSIDADE ESTADUAL DE CAMPINAS - UNICAMP. Tabela brasileira de composição de alimentos - TACO. 4. ed. Campinas: UNICAMP; NEPA, 2011.

WORD HEALTH ORGANIZATION - WHO. Reducing salt intake populations: report of a WHO forum and technical meeting. Paris, 2006. p. 23. 